\title{
INFLUÊNCIA DA RESOLUÇÃO ESPACIAL DE IMAGENS ORBITAIS NA IDENTIFICAÇÃO DE ELEMENTOS DA PAISAGEM EM ALTAMIRA-PA ${ }^{1}$
}

\author{
Flávio Jorge Ponzoni² e Ana Carolina Pinto Rezende ${ }^{2}$
}

\begin{abstract}
RESUMO - Imagens do sensor TM/Landsat-5 referentes às bandas de TM1 a TM5 e TM7 do município de AltamiraPA, Brasil, da passagem de 20/7/1991, modificadas para 60, 100, 120, 200 e $250 \mathrm{~m}$, foram utilizadas para avaliar a influência da resolução espacial na identificação de floresta, capoeira nova, capoeira madura e não-floresta. Mapas temáticos (um para cada resolução espacial) foram elaborados, considerando a aplicação de um algoritmo de classificação digital Bhattacharya (supervisionado), seguido da interpretação visual. A área de cada uma dessas categorias foi determinada em cada um dos mapas temáticos, tomando as imagens com $30 \mathrm{~m}$ de resolução espacial como referência. A exatidão de mapeamento foi avaliada, utilizando a exatidão global, o índice kappa e o índice Tau. Verificou-se que: a) as maiores discrepâncias em termos quantitativos ocorreram nos mapas gerados a partir das imagens com 200 m de resolução espacial; b) as categorias que dominavam a cena em termos espectrais e espaciais aumentaram à medida que a resolução espacial foi degradada; c) os mapas elaborados a partir de imagens com resolução espacial de 200 m apresentaram ligeira confusão na identificação dos temas; e d) a confusão espectral entre os temas não apresentou tendência linear com a gradativa degradação da resolução espectral.
\end{abstract}

Palavras-chave: Resolução espectral e mapeamento do uso da terra.

\section{INFLUENCE OF ORBITAL IMAGES SPATIAL RESOLUTION ON LANDSCAPE ELEMENT IDENTIFICATION IN ALTAMIRA, PA}

\begin{abstract}
TM/Landsat 5 images (TM1-TM5 and TM7 spectral bands) from Altamira PA, Brazil (07.20.1991) modified to 60,100,120, 200 and $250 \mathrm{~m}$ were utilized to evaluate the influence of the spatial resolution in the identification of Forest, Young secondary succession, Mature secondary succession and Non-forest. Thematic maps were made using the Bhattacharya (supervized) classification procedure, followed by visual interpretation. The area of each category was determined in each thematic map, using the $30 \mathrm{~m}$ spatial resolution as a reference. Mapping accuracy was evaluated using the Global accuracy, and the Kappa and Tau coefficients. It was found out that: a) the greatest area differences were observed in those maps derived from the $200 \mathrm{~m}$ spatial resolution images; b) those categories that dominated the scene spectrally and spatially, increased their influence as the spatial resolution decreased; c) the thematic maps from images with coarser spatial resolution than $200 \mathrm{~m}$ presented a slight confusion in map category identification, and d) the spectral confusion between the mapping categories did not present a linear tendency.
\end{abstract}

Key words: $\quad$ Spatial resolution, land-use mapping.

\section{INTRODUÇÃO}

Dentre os principais projetos de mapeamentos temáticos desenvolvidos no Brasil, destacam-se o da
Fundação S.O.S. Mata Atlântica (SOS), que junto com o Instituto Nacional de Pesquisas Espaciais (INPE) faz a atualização periódica de um atlas cuja legenda inclui remanescentes florestais, restinga e mangue existentes no

1 Recebido para publicação em 29.1.2002.

Aceito para publicação em 20.6.2002.

2 Instituto Nacional de Pesquisas Espaciais, Divisão de Sensoriamento Remoto, Avenida dos Astronautas, 1758, 12227-010 São José dos Campos-SP. 
território de dez Estados da Federação, e o do INPE, denominado Projeto Desflorestamentos na Amazônia (PRODES), que visa monitorar os desflorestamentos na Amazônia brasileira. Além destas iniciativas, existem outras sendo concebidas, que incluem o mapeamento de biomas como o cerrado e os campos sulinos. Em todos os casos, a vasta extensão geográfica requer o emprego de técnicas de sensoriamento remoto, realizado a partir da análise das imagens orbitais, cuja resolução espacial é um dos principais fatores na definição da legenda dos mapas temáticos.

A preocupação com os efeitos da resolução espacial nos trabalhos de mapeamento em andamento e, ou, sobre as futuras iniciativas vem aumentando devido à perspectiva de desenvolvimento de novos sensores orbitais dotados de diferentes capacidades. Dentre estes sensores destacam-se os do Satélite Sino-Brasileiro de Sensoriamento Remoto (CBERS) e aqueles planejados para os futuros satélites Brasileiro-Argentino-Espanhol (SABE) e o Satélite de Sensoriamento Remoto (SSR), os dois últimos ainda em fase de concepção.

Este trabalho, desenvolvido em Altamira (PA), tem como objetivo avaliar o efeito da variação da resolução espacial de imagens TM Landsat sobre a identificação/ quantificação de alguns elementos da paisagem em ambiente amazônico.

\section{MATERIAL E MÉTODOS}

\section{1. Área de Estudo}

Foi definida uma área de aproximadamente $45 \mathrm{x}$ $91 \mathrm{~km}$, estudada por Mausel et al. (1993), localizada ao longo da rodovia Transamazônica, na qual a vegetação dominante, segundo RADAMBRASIL (1974), é a Floresta Ombrófila Densa e a Floresta Ombrófila Aberta. A primeira, presente nos altos e baixos platôs, apresenta formações florestais bem uniformes, compostas por árvores grossas e altas, geralmente superiores a $50 \mathrm{~m}$, sem palmeiras e com raras lianas, nas quais está incluída a Floresta Ombrófila Submontana, caracterizada pela ocorrência de árvores baixas (de 10 a 15 m) a árvores altas (25 ou mais metros); a segunda apresenta-se com duas fisionomias predominantes: a floresta latifoliada e a mista. A floresta latifoliada, localizada em superfície aplainada, é bastante aberta, de baixa estatura e completamente coberta por lianas lenhosas; nas áreas mais acidentadas, é caracterizada por estreitos vales cobertos pelo babaçu e por largas encostas cobertas pelo cipoal, com árvores altas (com mais de $25 \mathrm{~m}$ ) e densamente distribuídas, embora as lianas continuem a envolver a maior parte da floresta. A floresta mista é constituída de palmeiras e árvores latifoliadas sempre verdes, bem espaçadas, de altura bastante irregular, apresentando grupamentos de babaçu nos vales rasos e concentrações de nanofoliadas deciduais nos testemunhos quartzolíticos das superfícies aplainadas.

A precipitação anual é de aproximadamente $1.700 \mathrm{~mm}$ em quatro meses úmidos, que ocorrem de junho a setembro (Mausel et al., 1993). A Figura 1 mostra a localização da área de estudo no contexto nacional.

A paisagem é caracterizada por uma rodovia principal (Rodovia Transamazônica) e por outras secundárias que se estendem pelo interior, percorrendo distâncias variadas. Ao longo dessa rede rodoviária são desenvolvidos diferentes tipos de agricultura, em que as pastagens constituem um uso típico do solo após o cultivo de diferentes culturas. As áreas abandonadas são comuns e caracterizadas por três estádios sucessionais da vegetação arbórea (Mausel et al.,1993): sucessão secundária inicial (SS1), caracterizada por uma cobertura vegetal graminóide, intercalada por elementos arbóreo/arbustivos; sucessão secundária intermediária (SS2), descrita como uma cobertura vegetal composta por árvores de 8 a $12 \mathrm{~m}$ de altura, mas desenvolvendo múltiplos estratos verticais, diferenciando-se de SS1 pela maior biomassa; e sucessão secundária avançada (SS3), apresentando também múltiplos estratos verticais maduros e árvores acima de $20 \mathrm{~m}$ de altura, muito similar a alguns tipos de florestas-clímax.

\subsection{Processamento Digital de Imagens}

As imagens TM/Landsat-5 das bandas espectrais de TM1 a TM5 e TM7 da órbita ponto 226/62 de 20/07/ 1991 (INPE,1994) foram georrefenciadas através de pontos de controle selecionados em carta topográfica na escala 1:100.000. Em seguida, aplicou-se o procedimento de simulação de imagens de baixa resolução espacial proposto por Banon (1990), obtendo-se novas imagens com as resoluções espaciais de 60, 100, 120, 200 e 250 m.

Os procedimentos adotados (modelo de mistura, segmentação e classificação) basearam-se na metodologia do Projeto PRODES, que se fundamenta na discriminação de áreas florestadas e não-florestadas na Região Amazônica. Segundo essa metodologia, aplica-se o modelo de mistura 
a todas as bandas espectrais para reduzir o número de imagens a serem tratadas digitalmente, gerando uma única imagem referente à componente sombra/água, sobre a qual são aplicados algoritmos de segmentação e de classificação digital (INPE, 2001). O modelo de mistura foi aplicado com a mesma função, porém, como os temas floresta, não-floresta, capoeira nova e capoeira madura não podem ser individualizados com o uso dessa única imagem (PRODES, 2001), a segmentação foi aplicada junto com as imagens das bandas espectrais 3, 4 e 5 de cada resolução espacial, inclusive a de $30 \mathrm{~m}$ para que os temas mencionados fossem detectáveis através dos algoritmos de segmentação e de classificação.

A segmentação foi aplicada com um limiar de separabilidade de 8 e tamanho de pixel de 50 , exceto para a resolução de $200 \mathrm{~m}$ e $250 \mathrm{~m}$, cujo limiar e tamanho de pixel foram de 5 e 15, respectivamente. Em seguida, procedeu-se à aplicação do algoritmo de classificação digital supervisionado Bhattacharya (INPE, 2001) para as imagens de cada resolução espacial, com um limiar de aceitação de $75 \%$, considerando somente as imagens de três bandas espectrais do sensor TM (bandas 3, 4 e 5). Os mapas temáticos resultantes foram submetidos à interpretação visual, para corrigir eventuais imperfeições.

\subsection{Comparação entre Mapas Temáticos e Cálculo de Exatidão}

A comparação entre os mapas temáticos fundamentou-se na quantificação dos temas da legenda. Primeiramente foram analisadas as quantidades de cada tema, determinadas em cada mapa temático. Em seguida, avaliou-se o grau de confusão encontrado entre os temas à medida que a resolução espacial foi degradada. Para tanto, assumiu-se o mapa temático gerado de imagens de resolução espacial de 30 m como referência, e os demais mapas foram então avaliados por meio da análise de matrizes de contingência, geradas da aplicação do procedimento de tabulação cruzada (INPE, 2001). Neste procedimento os mapas foram comparados dois a dois, sempre tendo como referência aquele gerado a partir da classificação das imagens de $30 \mathrm{~m}$ de resolução espacial. As matrizes de contingência apresentam seus elementos em hectares, sendo as linhas correspondentes às categorias da imagem de referência. Assim, foi possível avaliar o grau de coincidência entre os mapas temáticos, bem como o grau de confusão entre os temas, tendo sido gerados gráficos para facilitar a visualização dos resultados desse procedimento.

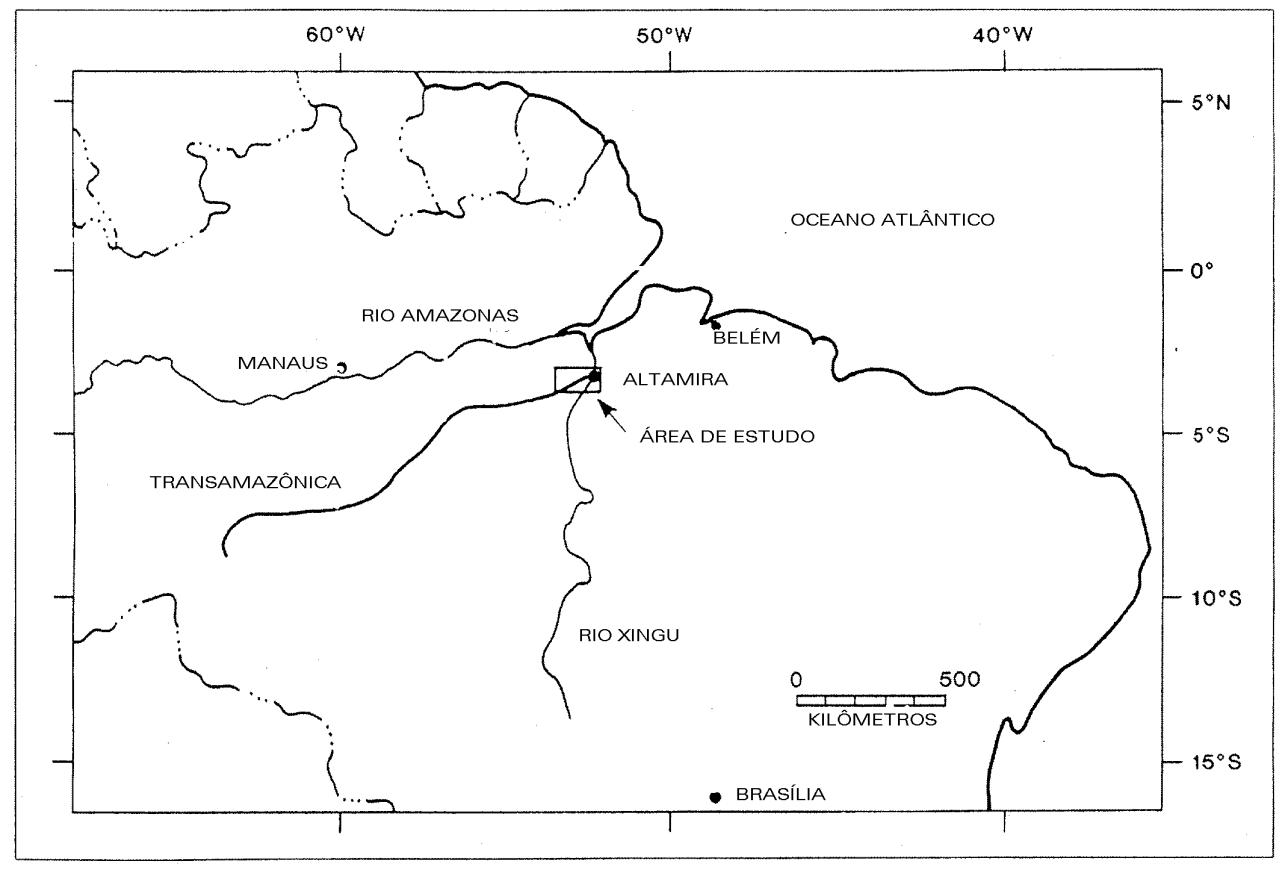

Figura 1 - Localização da área de estudo no contexto Nacional. Fonte: adaptada de Mausel et al. (1993). Figure 1 - Study area localization in the national context. Adapted from Mausel et al. (1993). 
Para cada uma dessas matrizes foram utilizados os índices de exatidão global, kappa e Tau (Ma e Redmond, 1995) e criados gráficos para melhor visualizar o comportamento dos três índices, à medida que se degradou a resolução espacial das imagens.

A exatidão global foi calculada através da equação:

$$
G=\frac{\sum_{i=1}^{M} n_{i i}}{n}
$$

em que $n_{i i}=$ elementos da diagonal da matriz de confusão; $n=$ número total de observações; e $M=$ número de temas mapeados.

O índice kappa foi calculado mediante a aplicação da seguinte equação:

$$
\text { Kappa }=\frac{n \sum_{i=1}^{M} x_{i}-\sum_{i=1}^{M}\left(x_{i+} x_{+i}\right)}{n^{2}-\sum_{i=1}^{M}\left(x_{i+} x_{+i}\right)}
$$

em que $n=$ número total de observações; $x_{i+}$ e $x_{+i}=$ somatório na linha e na coluna, respectivamente; e $M=$ número de temas mapeados.

Para o índice Tau, os cálculos foram feitos através das equações que se seguem:

$$
\begin{aligned}
& T=\frac{P_{0}-1 / M}{1-1 / M} \\
& \sigma_{T}^{2}=\frac{P_{0}\left(1-P_{0}\right)}{N(1-1 / M)^{2}}
\end{aligned}
$$

Os parâmetros $P_{0}$ e $P_{c}$ podem ser explicitadas por:

$$
\begin{gathered}
P_{0}=\frac{\sum_{i=1}^{M} n_{i i}}{N} \\
P_{c}=\frac{\sum_{i=1}^{M} n_{i+} n_{+i}}{N^{2}}
\end{gathered}
$$

R. Árvore, Viçosa-MG, v.26, n.4, p.403-410, 2002 em que $N=$ número total de pixels contemplados pela matriz de contingência; $n_{i i}=$ elementos da diagonal da matriz de contingência; $M=$ número de temas presentes na matriz de contingência; $P_{0}=$ representa a proporção de unidades que concordam; e $P_{c}=$ proporção de unidades que concordam por casualidade.

Segundo Brites et al. (1996), a exatidão global apresenta maiores valores em relação aos demais índices, pelo fato de considerar apenas a diagonal principal da matriz de contingência, ignorando a ocorrência de concordância casual entre as classes. Por outro lado, o coeficiente kappa, ao calcular a concordância casual, inclui nos cálculos os elementos da diagonal principal, fazendo com que esta seja superestimada, reduzindo o valor do índice. $\mathrm{Na}$ formulação do coeficiente Tau, o valor da concordância casual é estabelecido a priori, evitando as falhas em que incorrem a exatidão Global e o coeficiente kappa. Os valores intermediários de Tau indicam um bom balanceamento na consideração das concordâncias real e casual, tomando-se em consideração os dois extremos que representam os outros dois índices, ou seja, total desconsideração da casualidade e sua superestimativa. Tal fato é um indicador de que esse índice é o que representa com mais fidelidade a exatidão dos processos de classificação temática.

Outras razões que justificam o uso do coeficiente Tau, segundo Ma e Redmond, (1995), seriam: seu fácil entendimento e interpretação, uma vez que a concordância por chance $(\mathrm{Pc})$ é uma medida direta da classificação casual; o fato de prover clara indicação de como a classificação se comporta quando comparada com a identificação casual dos pixels às categorias ou classes; e o cálculo do índice e da estimativa de variância relativamente simples, uma vez que a concordância por chance é independente da matriz de contingência.

\section{RESULTADOS E DISCUSSÃO}

\subsection{Degradação das imagens e quantificação dos temas}

A elaboração de 30 imagens nas bandas espectrais de TM1 a TM5 e TM7, com resolução espacial de 60, $100,120,200$ e $250 \mathrm{~m}$, permitiu visualizar que a degradação espacial das imagens resultou na confusão de capoeiras nova com áreas de não-floresta e de capoeiras maduras com áreas de floresta, cuja dinâmica dos valores de reflectância de superfície para as capoeiras e as formações florestais clímax é apresentada na Figura 2. 


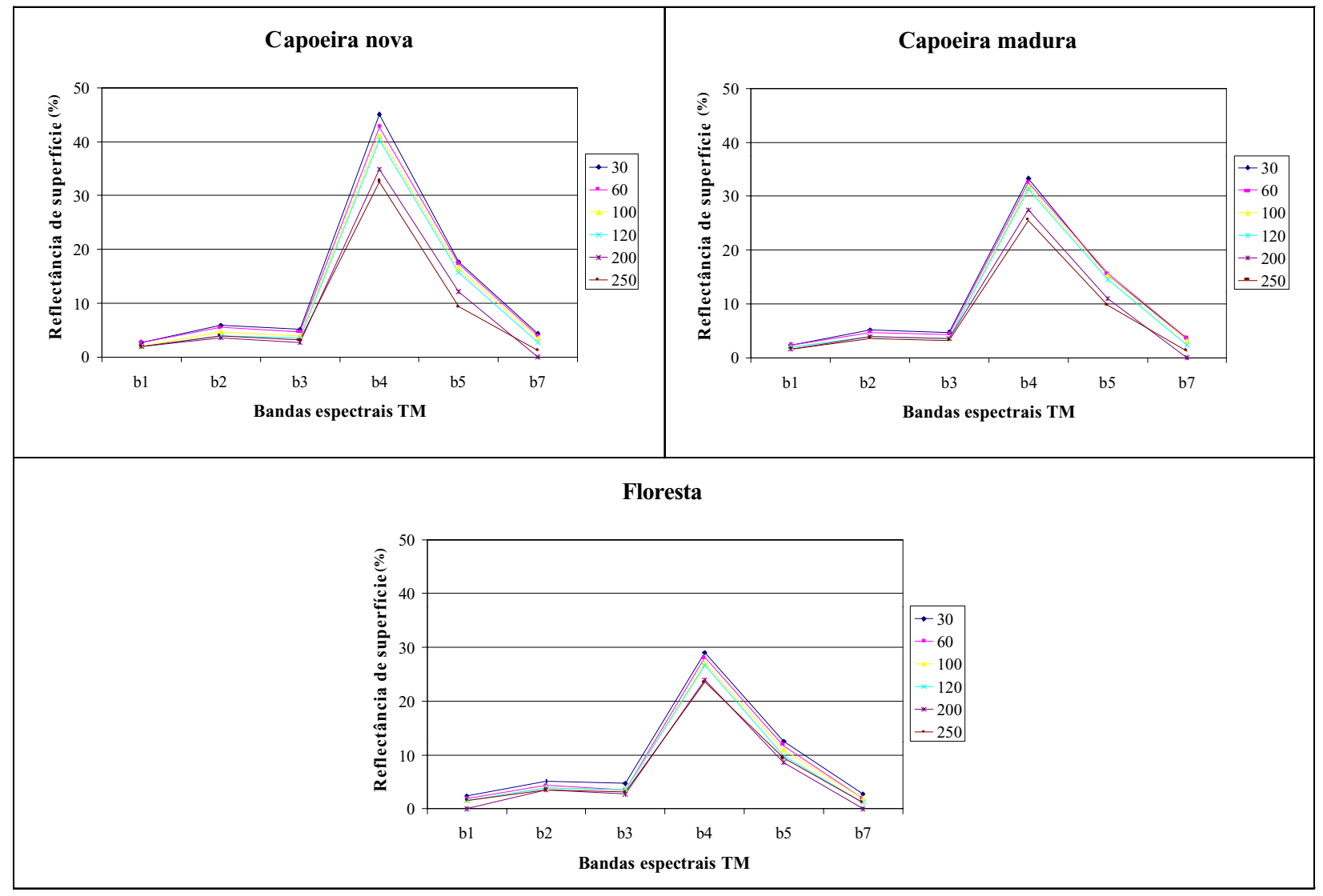

Figura 2 - Dinâmica das curvas de reflectância de superfície verificada nas formações arbóreas, mediante a degradação da resolução espacial.

Figure 2 - Surface reflectance dynamic observed in the woody vegetation through the spatial resolution decrease.

Observa-se que tanto as capoeiras como a floresta apresentaram valores de reflectância de superfície decrescentes com a diminuição da resolução espacial das imagens, em toda a região espectral considerada. Tal fato significa que houve tendência de homogeneizar a cena como um todo, fazendo com que as formações arbóreas se assemelhem espectralmente entre si à medida que a resolução espacial foi degradada.

A Figura 3 apresenta a dinâmica das áreas dos temas considerados.

Observa-se que a área do tema floresta apresentou tendência de queda nas simulações de 60 e 100 m, e de subida nas demais simulações. A correlação inversa existente entre os temas floresta e não-floresta pode ser explicada pelo fato de, com a degradação da resolução espacial, o tema dominante na cena tender a se expandir, englobando aqueles mais parecidos espectralmente

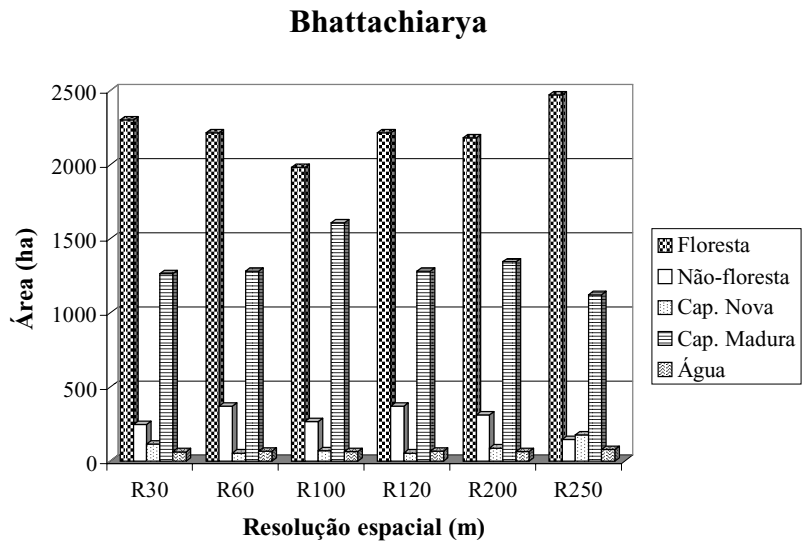

Figura 3 - Áreas dos temas originadas da aplicação dos algoritmos Isoseg e Bhattacharya em diferentes resoluções espaciais.

Figure 3-Areas of the mapping categories from both Isoseg and Battacharya classification procedures and different spatial resolutions.

R. Árvore, Viçosa-MG, v.26, n.4, p.403-410, 2002 
(Ponzoni et al., 2001). O tema não-floresta apresentou ainda tendência de diminuir sua área nos mapas elaborados a partir da resolução de $250 \mathrm{~m}$. A capoeira nova apresentou correlação com o tema não-floresta, assim como a capoeira madura com a floresta.

Para melhor compreender as relações entre esses temas, apresentam-se os resultados das matrizes de contingência geradas dos diferentes mapas temáticos cruzados dois a dois, tendo como referência o de resolução espacial de $30 \mathrm{~m}$ (Figura 4). Exemplificando, na coluna 1 de um determinado nível de resolução espacial, os padrões que eventualmente aparecem referem-se aos demais temas que foram confundidos com o tema floresta em relação ao mapa temático de $30 \mathrm{~m}$. Vale salientar que as cores aparecem na mesma seqüência, ou seja, 1-floresta, 2-nãofloresta, 3-capoeira nova, 4-capoeira madura e da 5-água, dependendo da ocorrência ou não de confusão.

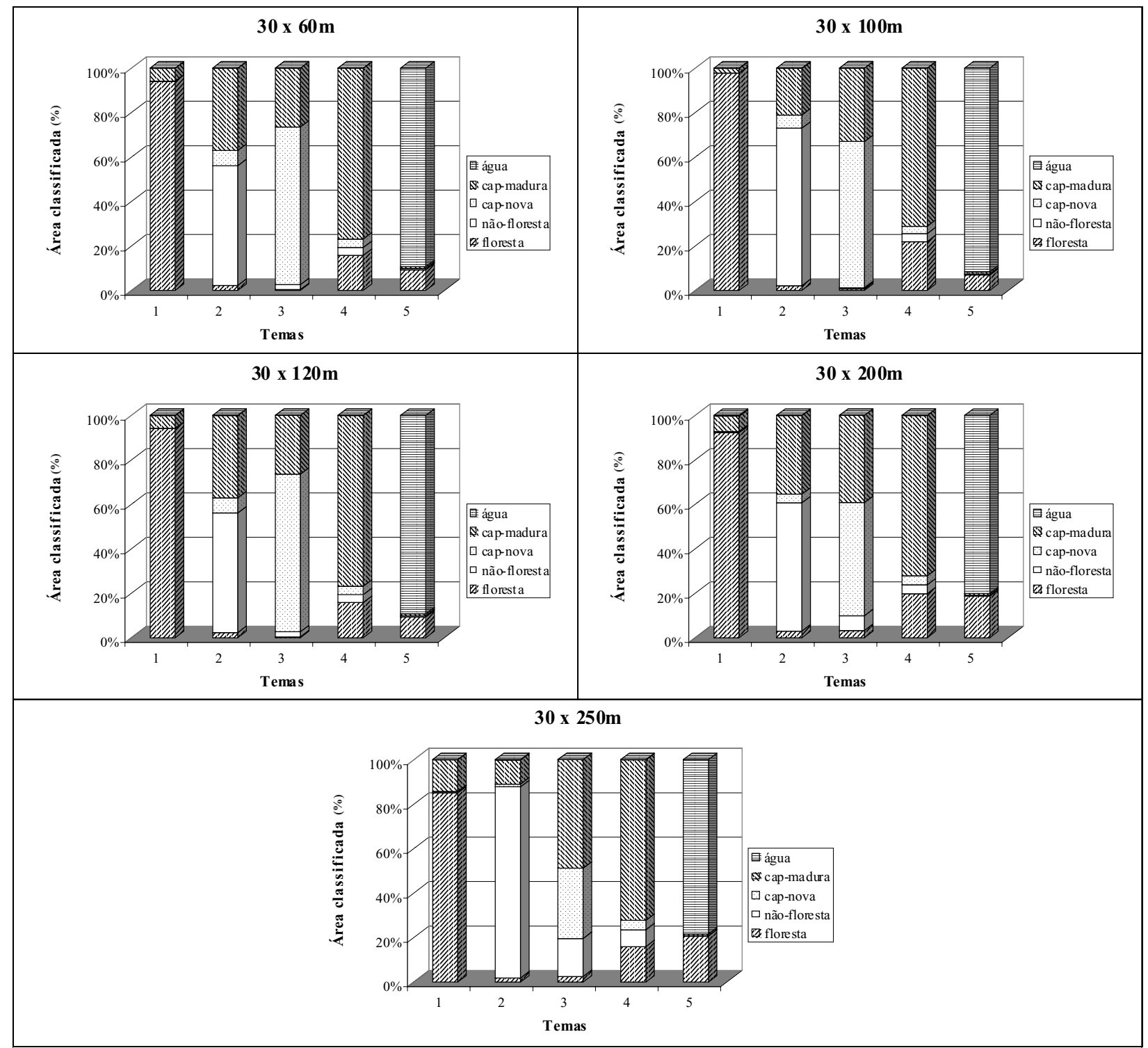

Figura 4 - Gráficos gerados a partir dos dados das matrizes de contingências.

Figure 4-Graphics showing the error matrices data. 
O tema floresta foi sempre confundido com o tema capoeira madura em proporções relativamente constantes, a não ser na simulação de $250 \mathrm{~m}$, quando ocorreu um ligeiro aumento da confusão entre eles. $O$ tema não-floresta confundiu-se com as capoeiras, destacando-se que a diminuição da confusão entre eles na simulação de $250 \mathrm{~m}$ pode ser atribuída à diminuição de área classificada como capoeiras.

Os temas capoeira nova e capoeira madura, que apresentaram grande confusão entre si, experimentaram leve declínio à medida que a resolução espacial foi sendo degradada. $\mathrm{O}$ aumento da confusão com o tema nãofloresta na simulação de $250 \mathrm{~m}$ pode ser atribuído à tendência de "desaparecimento" do tema capoeira nova da cena, à medida que a resolução espacial foi sendo degradada. Surpreendentemente, o tema água também apresentou índices de confusão com os temas floresta e capoeira madura, provavelmente provocados pelo "desaparecimento" de pequenos corpos d'água ao longo de toda a cena.

\subsection{Exatidão de Mapeamento}

Os valores da exatidão global $(\mathrm{G})$, índice kappa $(\mathrm{K})$ e índice Tau (T) estão apresentados no Quadro 1 e na Figura 5.

Observa-se que os valores de $\mathrm{G}$ foram sempre superiores aos demais índices de exatidão, confirmando a observações de Brites et al. (1996) sobre a tendência de esse índice superestimar a exatidão de mapeamento. $\mathrm{O}$ índice Tau resulta em valores intermediários entre $\mathrm{G}$ e $K$. De qualquer forma, à medida que as imagens tiveram

Quadro 1 - Exatidão Global (G), índice kappa (K) e índice Tau (T)

Table 1 - Global accuracy (G), Kappa coefficient (K) and Tau Coefficient (T)

\begin{tabular}{|l|c|c|c|}
\hline \multirow{2}{*}{ Comparação } & \multicolumn{3}{|c|}{ Bhattacharya } \\
\cline { 2 - 4 } & $\mathrm{G}$ & $\mathrm{K}$ & $\mathrm{T}$ \\
\hline $30 \times 60 \mathrm{~m}$ & 0,84 & 0,73 & 0,81 \\
$30 \times 100 \mathrm{~m}$ & 0,85 & 0,74 & 0,81 \\
$30 \times 120 \mathrm{~m}$ & 0,84 & 0,73 & 0,81 \\
$30 \times 200 \mathrm{~m}$ & 0,82 & 0,68 & 0,78 \\
$30 \times 250 \mathrm{~m}$ & 0,79 & 0,62 & 0,75 \\
\hline
\end{tabular}

sua resolução espacial degradada os índices apresentaram queda, acentuando-se a partir de $200 \mathrm{~m}$.

\section{Exatidão para Bhattacharya}

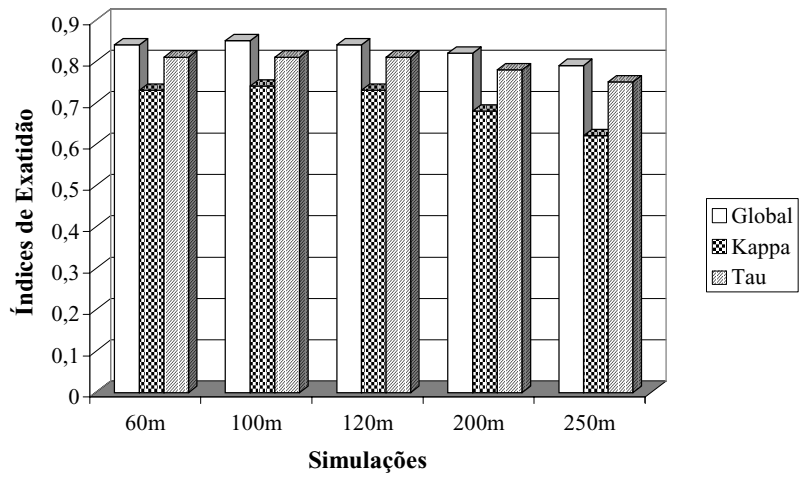

Figura 5 - Representação gráfica dos índices de exatidão global, coeficiente kappa e Tau.

Figure 5 - Accuracy mapping coefficients graphic representation.

\section{CONCLUSÕES}

A partir dos resultados alcançados, conclui-se que as alterações mais significativas ocorreram com imagens de resolução espacial igual ou superior a $200 \mathrm{~m}$.

Os temas com semelhança espectral apresentaram maior grau de confusão entre si, ao se promover a degradação da resolução espacial, cuja confusão não foi gradativa entre os níveis de resolução espacial adotados. Ainda, os índices de exatidão de mapeamento indicaram que a identificação dos temas foi comprometida quando foram adotadas imagens com resolução espacial com pixel de dimensões superiores a $200 \mathrm{~m}$.

\section{AGRADECIMENTO}

Ao Anthropological Center for Trainning and Research on Global Environmental Change da Indiana University (ACT/IU), pela disponibilidade de recursos financeiros e de dados que viabilizaram a realização deste trabalho.

\section{REFERÊNCIAS BIBLIOGRÁFICAS}

BANON, G. J. F. Simulação de imagens de baixa resolução. Revista da Sociedade Brasileira de Automática, v. 2 n. 3, p. 180-192, 1990 .

R. Árvore, Viçosa-MG, v.26, n.4, p.403-410, 2002 
BRITES, R. S.; SOARES, V. P.; RIBEIRO, C. A. A. S. Comparação de desempenho entre três índices de exatidão aplicados a classificações de imagens orbitais. In: SIMPÓSIO BRASILEIRO DE SENSORIAMENTO REMOTO, 8, 1996, Salvador. Anais... São Paulo: Image Multimídia, 1996. Seção de Comunicações Técnico Científicas (CD-ROM).

INSTITUTO DE PESQUISA ESPACIAL - INPE. 2001. Disponível em: http://www.dpi.inpe.br/spring/portugues/ index.html

INSTITUTO DE PESQUISA ESPACIAL - INPE. Imagem de satélite Landsat TM -ORB 226 P 062 . [CD ROM]. Data Pass. 20-07-1991. Cachoeira Paulista: Divisão de Geração de Imagens (DGI), 1994.

MA, Z.; REDMOND, R. L. Tau coefficient for accuracy assessment of classification of remote sensing data.

Photogrammetric Engineering and Remote Sensing, v. 61, n. 4, p. 435-439, 1995.
MAUSEL, P. et al. Espectral Identification of successional Stages following Deforestation in the Amazon. Geocarto International, v. 8, n. 4, p. 61-81, 1993.

PONZONI, F. J.; GALVÃO, L. S.; EPIPHANIO, J. C. N. Influência da resolução espacial sobre a quantificação de áreas não florestadas em ambiente amazônico. In: SIMPÓSIO BRASILEIRO DE SENSORIAMENTO REMOTO, 10, 2001, Foz do Iguaçu. Anais... São Paulo: Sonopress, 2001. (CD-ROM).

PRODES. Monitoramento da Floresta Amazônica Por Satélite. Instituto Nacional de Pesquisas Espaciais. Disponível em: http://sputnik.dpi.inpe.br:1910/col/ dpi.inpe.br/banon/2000/09.12.17.24/doc/amz1998_1999/ index_amz.htm,2001

PROJETO RADAM BRASIL. Folha SA.22 Belém Geologia, Geomorfologia, Solos, Vegetação e Uso Potencial da Terra. Rio de Janeiro: 1974. (Levantamento de recursos Naturais, 5). 